\title{
On the closed range problem for composition operators on the Dirichlet space
}

https://doi.org/10.1515/conop-2019-0007

Received October 19, 2018; accepted June 12, 2019

Abstract: We characterize closed range composition operators on the Dirichlet space for a particular class of composition symbols. The characterization relies on a result about Fredholm Toeplitz operators with $B M O^{1}$ symbols, and with Berezin transforms of vanishing oscillation.

Keywords: composition operator, Dirichlet space, Toeplitz operator, Berezin transform, closed range operator, vanishing mean oscillation.

MSC: 47B33, 47A53, 30H25, 30H35, 30J10

This paper, although largely of expository nature, provides also a new partial result on a long standing open problem of characterizing the closed range composition operators on the Dirichlet space. Moreover, while investigating what is seemingly a very particular property of this specific class of operators, we also show the connections to some other interesting topics, and state a few related problems.

Composition operators are an important, natural class of examples of specific operators on spaces of holomorphic functions. Exploring the properties of such operators provides, in general, interesting connections between different areas of mathematics. In this paper we give a closed range characterization for a class of composition operators on the Dirichlet space, by using the Berezin transform as a specific tool of this exploration. We also point out the underlying connections to the maximal ideal space of $H^{\infty}$, and the use of the Berezin transform behaviour on its Gleason parts. Similar connection have already been realized and used in the past such as, for example, in the results obtained in [11], [6], [12], [3] and [4]. Our contributions here include showing further such connections, and providing some interesting related questions for future exploration.

We start with some basic notation and few definitions.

Let $\mathbb{D}$ denote the open unit disk in the complex plane, and let $\mathcal{H}(\mathbb{D})$ be the space of holomorphic, complex valued functions on $\mathbb{D}$. For $z \in \mathbb{D}$,

$$
\psi_{z}(w)=\frac{z-w}{1-\bar{z} w}
$$

is the involutive automorphism of $\mathbb{D}$ mapping 0 into $z$. The pseudo-hyperbolic metric $\rho$ on $\mathbb{D}$ is defined by $\rho(z, w)=\left|\psi_{z}(w)\right|$, and the hyperbolic metric $\beta$ on $\mathbb{D}$ is defined by

$$
\beta(z, w)=\tanh ^{-1} \rho(z, w) .
$$

The Bergman space $L_{a}^{2}(\mathbb{D})$ is a Hilbert space of holomorphic functions defined by

$$
L_{a}^{2}(\mathbb{D})=\left\{f \in \mathcal{H}(\mathbb{D}) ;\|f\|^{2}=\int_{\mathbb{D}}|f(z)|^{2} d m(z)<\infty\right\},
$$

`Corresponding Author: Nina Zorboska : Department of Mathematics, University of Manitoba, Winnipeg, MB, R3T 2N2, Canada, E-mail: Nina.Zorboska@umanitoba.ca 
where $\mathrm{dm}$ stands for the normalized Lebesgue area measure on the disk. The Bergman space is a reproducing kernel Hilbert space with normalized point evaluation functions given by

$$
k_{z}(w)=\frac{\left(1-|z|^{2}\right)}{(1-\bar{z} w)^{2}} .
$$

The Dirichlet space $\mathcal{D}(\mathbb{D})$ is the Hilbert space of holomorphic functions whose derivatives are in the Bergman space. The Dirichlet norm for $f \in \mathcal{D}(\mathbb{D})$ is defined by

$$
\|f\|_{\mathcal{D}}^{2}=|f(0)|^{2}+\int_{\mathbb{D}}\left|f^{\prime}(z)\right|^{2} d m(z) .
$$

One of the long standing open problems about specific operators on spaces of holomorphic functions is determining the composition operators on the Dirichlet space that have a closed range.

For a non-constant holomorphic function $\phi$ mapping $\mathbb{D}$ into itself, the composition operator induced by the symbol $\phi$ is defined on $\mathcal{H}(\mathbb{D})$ by

$$
C_{\phi} f=f \circ \phi .
$$

The boundedness and the compactness of composition operators on the Dirichlet space have been determined in several different ways, using slightly different approaches and methods. The methods closest to the ones we will use here appear, for example, in [1] and [16], and we refer the reader to these sources for more details.

The closed range problem for composition operators on the Dirichlet space has been explored in several papers (see for example [7], [10], [5]). The complete characterization is known in the case when the inducing map is of bounded multiplicity (see [7]), but the general problem is still open. In most instances, solving a problem about composition operators on the Dirichlet space is done by investigating a corresponding problem about related Toeplitz operators on the Bergman space. We follow this approach here for exploring the closedness of the range of a specific class of composition operators.

Before we are ready to state our result, we need to briefly mention few details of the general set-up, and recall few more definitions.

For a measurable $f$ in $L^{1}(\mathbb{D})$, the Toeplitz operator $T_{f}$ on $L_{a}^{2}(\mathbb{D})$ is defined by

$$
T_{f} g(z)=P(f g)(z)=\int_{\mathbb{D}} f(w) g(w) \frac{1}{(1-z \bar{w})^{2}} d m(w),
$$

where $P$ stands for the Bergman projection, i.e. $P$ is the orthogonal projection of $L^{2}(\mathbb{D})$ onto $L_{a}^{2}(\mathbb{D})$.

The Berezin transform of $T_{f}$ (and of $f$ ) on $L_{a}^{2}(\mathbb{D})$ is defined by

$$
\widetilde{T_{f}}(z)=\widetilde{f}(z)=<T_{f} k_{z}, k_{z}>=\int_{\mathbb{D}} f(w) \frac{\left(1-|z|^{2}\right)^{2}}{|1-z \bar{w}|^{4}} d m(w) .
$$

The Berezin transform can be defined for any operator on a reproducing kernel Hilbert space of holomorphic functions, and it is a continuous function that uniquely determines the operator. Thus, it is natural to expect that some of the properties of the operator can be characterized by a specific behaviour of their Berezin transform, and we employ this method here, when trying to classify the closed range composition operators on the Dirichlet space.

Without loss of generality for the closed range problem for $C_{\phi}$ on $\mathcal{D}(\mathbb{D})$, from now on we will assume that $\phi(0)=0$. This is possible since any $C_{\psi_{a}}$, with $\psi_{a}$ a disk automorphism defined above, is an invertible operator on $\mathcal{D}(\mathbb{D})$, i.e. it has a closed range. Moreover, note that if $\phi(0)=a$, then $\psi_{a} \circ \phi(0)=0$.

For (a non-constant, holomorphic) self-map $\phi$ of $\mathbb{D}$, define $n_{\phi}$ to be the counting function for $\phi$, namely $n_{\phi}(w)$ equals the size of the set $\phi^{-1}(\{w\})$, which is at most countably infinite. 
By the change of variable formula, for any $f \in \mathcal{D}(\mathbb{D})$ we have that

$$
\left\|C_{\phi} f\right\|_{\mathcal{D}}^{2}=|f(0)|^{2}+\int_{\mathbb{D}}\left|f^{\prime}(w)\right|^{2} n_{\phi}(w) d m(w)=|f(0)|^{2}+<T_{n_{\phi}} f^{\prime}, f^{\prime}>,
$$

where $f^{\prime} \in L_{a}^{2}(\mathbb{D}), T_{n_{\phi}}$ is a Toeplitz operator on $L_{a}^{2}(\mathbb{D})$, and the inner product is the inner product in $L_{a}^{2}(\mathbb{D})$.

Here are two more fairly trivial facts:

- The differentiation operator $D(f)=f^{\prime}$ is an isometric isomorphism between $\mathcal{D}_{0}(\mathbb{D})=\{f \in \mathcal{D}(\mathbb{D}): f(0)=0\}$ and $L_{a}^{2}(\mathbb{D})$.

- $C_{\phi} 1=1$, and $C_{\phi}\left(\mathcal{D}_{0}(\mathbb{D})\right) \subset \mathcal{D}_{0}(\mathbb{D})$.

Thus, using the equation ( $\left.{ }^{\star}\right)$ from above, it is easy to conclude that:

- $C_{\phi}$ is bounded, or compact, on $\mathcal{D}(\mathbb{D})$ if and only if $T_{n_{\phi}}$ is bounded, or compact, on $L_{a}^{2}(\mathbb{D})$.

Since $n_{\phi}(w) \geq 0$ on $\mathbb{D}, T_{n_{\phi}}$ is a positive operator, and so $T_{n_{\phi}}$ is bounded (or compact) on $L_{a}^{2}(\mathbb{D})$ if and only if $\widetilde{n_{\phi}}$, the Berezin transform of $n_{\phi}$, is bounded on $\mathbb{D}$ (or $\widetilde{n_{\phi}}(z) \rightarrow 0$, as $|z| \rightarrow 1$, correspondingly). For details on this results and more, see for example [15].

As for the connections between the closed range problem of $C_{\phi}$ on $\mathcal{D}(\mathbb{D})$ and the corresponding properties of the Toeplitz operator $T_{n_{\phi}}$ on $L_{a}^{2}(\mathbb{D})$, we first recall a few basic complex analysis and operator theoretic results.

From the fact that a non-constant holomorphic function $\phi$ is an open map, we can easily conclude that $\operatorname{Ker}\left(C_{\phi}\right)=\{0\}$. It is a standard operator theory result that then $C_{\phi}$ has a closed range on $\mathcal{D}(\mathbb{D})$ if and only if $C_{\phi}$ is bounded below, which is furthermore equivalent to $C_{\phi}^{\star} C_{\phi}$ being invertible. From the equation (*) and the discussion above, we can see that this is also equivalent to the Toeplitz operator $T_{n_{\phi}}$ being invertible on $L_{a}^{2}(\mathbb{D})$, i.e.

- $C_{\phi}$ has a closed range on $\mathcal{D}(\mathbb{D})$ if and only if $T_{n_{\phi}}$ is invertible on $L_{a}^{2}(\mathbb{D})$.

It is also known that if $n_{\phi}$ is bounded on $\mathbb{D}$, i.e. if $\phi$ is of bounded multiplicity (valence), then $T_{n_{\phi}}$ is invertible on $L_{a}^{2}(\mathbb{D})$ if and only if $\widetilde{n_{\phi}}$ is bounded away from zero (see [8], [13]). Hence, as was already mentioned above

- If $\phi$ is of bounded multiplicity, $C_{\phi}$ has a closed range on $\mathcal{D}(\mathbb{D})$ if and only if $\widetilde{n_{\phi}}$ is bounded away from zero.

On the other hand, if $\phi$ is a full map, i.e $n_{\phi}(w) \geq 1$ almost everywhere on $\mathbb{D}$, it is again easy to see from the equation ( $\left.{ }^{\star}\right)$ above that $C_{\phi}$ is bounded below on $\mathcal{D}(\mathbb{D})$, i.e. that $C_{\phi}$ has a closed range. Thus, the interesting cases lie somewhere in between, namely when $\phi$ is of unbounded multiplicity and $\phi$ is not a full map.

An example provided in [10] shows that when $\widetilde{n_{\phi}}$ is bounded and $\phi$ is of unbounded multiplicity, i.e. when $n_{\phi}$ is not bounded on $\mathbb{D}$, the boundedness from below (away from zero) of $\widetilde{n_{\phi}}$ does not guarantee that $C_{\phi}$ has a closed range on $\mathcal{D}(\mathbb{D})$.

Using a recent result from [18] about Toeplitz operators with possibly unbounded symbols and their Fredholmness on the Bergman space, we will provide a characterization of closed range composition operators $C_{\phi}$ on the Dirichlet space for a class of maps $\phi$ with possibly unbounded multiplicity. In order to accomplish this, we have to introduce few more concepts.

Recall that for $f \in L^{1}(\mathbb{D})$ and $p \geq 1$ we say that $f \in B M O^{p}$ if

$$
\|f\|_{B M O^{p}}^{p}=\sup _{z \in \mathbb{D}}\left(\widetilde{|f|^{p}}(z)-|\widetilde{f}|^{p}(z)\right)<\infty,
$$

and $f \in V M O^{p}$ if

$$
\left.\lim _{|z| \rightarrow 1} \widetilde{\left.|| f\right|^{p}}(z)-|\widetilde{f}|^{p}(z)\right) \rightarrow 0
$$


The $B M O^{p}$ spaces are Banach spaces satisfying

$$
L^{\infty} \subset B M O^{p} \subset B M O^{q} \subset B M O^{1},
$$

for $1<q<p$. Similar inclusions, except for the first one, also hold for the $V M O^{p}$ spaces. Note that $B M O$ stands for "bounded mean oscillation", and VMO stands for "vanishing mean oscillation".

The space of functions of vanishing oscillation on the unit disk is defined by

$$
V O=\{f \in C(\mathbb{D}): \sup \{|f(z)-f(w)|: w \in D(z, 1 / 2)\} \rightarrow 0,|z| \rightarrow 1\} .
$$

Note that

$$
C(\overline{\mathbb{D}}) \subset V O \subset V M O^{2} \text {, and } V O \subset U C_{\beta}(\mathbb{D}),
$$

where $U C_{\beta}(\mathbb{D})$ denotes the space of uniformly continuous, with respect to the Bergman metric $\beta$, functions on $\mathbb{D}$.

For more details on the $B M O^{p}, V M O^{p}$ and $V O$ spaces see [14], [15] and [9].

When considering Toeplitz operators on the Bergman space via the Berezin transform, the $B M O^{1}$ space provides a natural class of possible unbounded symbols, as was shown in the following result.

Theorem 1. [17]: Let $f \in B M O^{1}$. Then $T_{f}$ is bounded on $L_{a}^{2}(\mathbb{D})$ if and only if $\tilde{f}$ is bounded on $\mathbb{D}$, and $T_{f}$ is compact if and only if $\widetilde{f}(z) \rightarrow 0$, as $|z| \rightarrow 1$.

Remark 1. It is known that if $f \in B M O^{1}$ and $\widetilde{f} \in L^{\infty}$, then $\widetilde{f}$ is Lipschitz with respect to the Bergman metric $\beta$ (see [15]), and so $\widetilde{f} \in L^{\infty} \cap \operatorname{Lip}_{\beta}(\mathbb{D}) \subset B U C_{\beta}(\mathbb{D})$, where $\operatorname{Lip}_{\beta}(\mathbb{D})$ and $B U C_{\beta}(\mathbb{D})$ denote the Lipschitz, and the bounded uniformly continuous, functions with respect to the Bergman metric $\beta$ on $\mathbb{D}$.

As it was mentioned before, the Berezin transform function $\widetilde{f}$ is always in $C(\mathbb{D})$. Assuming that $\tilde{f}$ is also in $L^{\infty}$, and since $\mathbb{D}$ is dense in $\mathfrak{M}$, the maximal ideal space of $H^{\infty}$, one can ask if $\widetilde{f}$ can be continuously extended to $\mathfrak{M}$, or possibly to some parts of $\mathfrak{M}$. Problems like this have been considered in [4] for Berezin symbols of operators in the Toeplitz algebra, and in [2] for general functions in $B U C_{\beta}(\mathbb{D})$.

Recall that the Toeplitz algebra $\mathcal{T}$ is the closed subalgebra of $\mathcal{B}\left(L_{a}^{2}(\mathbb{D})\right)$ generated by $\left\{T_{f}: f \in L^{\infty}\right\}$. A result in [4, Theorem 2.11] says that if $T \in \mathcal{T}$, then $\widetilde{T} \in C(\mathfrak{M})$, i.e. $\widetilde{T}$ can be continuously extended to all of $\mathfrak{M}$.

Checking if a specific operator is in the Toeplitz algebra $\mathcal{T}$ or not, is in general not an easy task, and there aren't too many results of this kind in the literature. Same is true for determining if a specific (non harmonic) bounded function in $C(\mathbb{D})$ can be extended continuously to all of $\mathfrak{M}$.

Interestingly enough, as we will see below, the type of conditions we require on the Berezin transform $\widetilde{n_{\phi}}$ when determining the closed range composition operators on the Dirichlet space, also imply that $\widetilde{n_{\phi}}$ can be continuously extended to $\mathfrak{M}$. We will state few other related remarks and questions below, after Theorem 3 , but let us first give the last piece of background needed to show our main result. $\diamond$

Recall that an operator is Fredholm whenever it is invertible modulo the compact operators, or equivalently, if and only if its kernel and co-kernel are finite dimensional. This immediately implies that the Fredholm operators also have a closed range. There are several results characterizing the Fredholmness of Toeplitz operators on the Bergman space with special type of symbols, most of them requiring that the symbol is bounded, or that it is continuous.

The following characterization of Fredholm Toeplitz operators $T_{f}$ with $f$ in $B M O^{1}$, was obtained in [18].

Theorem 2. [18]: Let $f \in B M O^{1}$ and let $\tilde{f} \in L^{\infty}$, so that $T_{f}$ is bounded on $L_{a}^{2}(\mathbb{D})$. If $\widetilde{f} \in V O$, then

(i) $T_{f}$ is Fredholm if and only if $\exists \delta>0, \exists r \in(0,1)$ such that $|\widetilde{f}(z)| \geq \delta$, whenever $|z| \geq r$.

(ii) $T_{f}$ is in the Toeplitz algebra $\mathcal{T}$, and so $\tilde{f} \in C(\mathfrak{M})$.

Using Theorem 2 above, we have the following result on closed range composition operators on the Dirichlet space, which includes cases when the symbol $\phi$ is possibly of unbounded multiplicity.

Theorem 3. Let $\phi$ be a non-constant, holomorphic self-map of $\mathbb{D}$ such that the composition operator $C_{\phi}$ is bounded on $\mathcal{D}(\mathbb{D})$. If $\widetilde{n_{\phi}} \in V O$, then $C_{\phi}$ has a closed range on $\mathcal{D}(\mathbb{D})$ if and only if $\exists \delta>0, \exists r \in(0,1)$ such that $\widetilde{n_{\phi}}(w) \geq \delta$, whenever $|w| \geq r$. 
Proof. Since $n_{\phi} \geq 0$ on $\mathbb{D}, n_{\phi} \in B M O^{1}$. Also, from the equation ( $\left.{ }^{\star}\right)$ above, the composition operator $C_{\phi}$ is bounded on $\mathcal{D}(\mathbb{D})$ if and only if the Toeplitz operator $T_{n_{\phi}}$ is bounded on $L_{a}^{2}(\mathbb{D})$, which is further equivalent to $\widetilde{n_{\phi}}$ bounded on $\mathbb{D}$. Thus, by Theorem 2, part (i), if $\widetilde{n_{\phi}} \in V O$, then $T_{n_{\phi}}$ is Fredholm if and only if $\exists \delta>0$, $\exists r \in(0,1)$ such that $\widetilde{n_{\phi}}(w) \geq \delta$, whenever $|w| \geq r$.

The Toeplitz operator $T_{n_{\phi}}$ is a positive operator, and so it is also a selfadjoint operator. Hence, if $T_{n_{\phi}}$ is Fredholm, it must have index zero. Furthermore, since $\phi(\mathbb{D})$ is an open (non-empty) subset of $\mathbb{D}$ and $n_{\phi}(w) \geq 1$ for every $w \in \phi(\mathbb{D})$, for any $g \in L_{a}^{2}(\mathbb{D})$ we have that

$$
\left\langle T_{n_{\phi}} g, g>=\int_{\mathbb{D}}|g(w)|^{2} n_{\phi}(w) d m(w) \geq \int_{\phi(\mathbb{D})}|g(w)|^{2} d m(w) .\right.
$$

This implies that if $g \in \operatorname{Ker}\left(T_{n_{\phi}}\right)$, then $g$ is almost everywhere zero on $\phi(\mathbb{D})$. But $g \in \mathcal{H}(\mathbb{D}), \phi(\mathbb{D})$ is open subset of $\mathbb{D}$, and so $g$ must be zero. Hence, the kernel of $T_{n_{\phi}}$ must be trivial.

Thus, $T_{n_{\phi}}$ is Fredholm on $L_{a}^{2}(\mathbb{D})$ if and only if it is invertible, which is furthermore equivalent to $C_{\phi}$ being bounded below on $\mathcal{D}_{0}(\mathbb{D})$, i.e equivalent to $C_{\phi}$ having a closed range on $\mathcal{D}(\mathbb{D})$.

Remark 2. Note that, in fact, $\widetilde{n_{\phi}}(w)>0$, for any $w \in \mathbb{D}$. This is true since when $\phi$ is not a constant function, $\phi(\mathbb{D})$ is an open subset of $\mathbb{D}$, and so, similarly as above, using that $k_{w} \in \mathcal{H}(\mathbb{D})$ we get that

$$
\widetilde{n_{\phi}}(w)=\int_{\mathbb{D}} n_{\phi}(z)\left|k_{w}(z)\right|^{2} d m(z) \geq \int_{\phi(\mathbb{D})}\left|k_{w}(z)\right|^{2} d m(z)>0 .
$$

Since $\widetilde{n_{\phi}}$ is also in $C(\mathbb{D})$, this shows that the boundedness from below (away from zero) of $\widetilde{n_{\phi}}(w)$ for $|w| \geq r>0$, implies that there exists $\delta_{1}>0$ such that $\widetilde{n_{\phi}}(w) \geq \delta_{1}$ for all $w$ in $\mathbb{D}$, i.e. that $\widetilde{n_{\phi}}$ is bounded below on all of $\mathbb{D}$.

Thus, for $\widetilde{n_{\phi}} \in L^{\infty} \cap V O, C_{\phi}$ has a closed range on $\mathcal{D}(\mathbb{D})$ if and only if $\exists \delta>0$, such that $\widetilde{n_{\phi}}(w) \geq \delta, \forall w \in \mathbb{D}$. $\diamond$

A natural problem that follows from the above theorem is to determine for which holomorphic nonconstant self-maps $\phi$ of $\mathbb{D}$, does $\widetilde{n_{\phi}} \in L^{\infty} \cap V O$. For example, one necessary condition is that $\phi \in \mathcal{D}(\mathbb{D})$, but this condition is not sufficient even to have that $\widetilde{n_{\phi}} \in L^{\infty}$.

From Theorem 2, part (ii), we also see that if $\widetilde{n_{\phi}} \in L^{\infty} \cap V O$, then $\widetilde{n_{\phi}} \in C(\mathfrak{M})$. Thus, we can use a result from [12, Theorem 30] which says that then $\widetilde{n_{\phi}} \in V O$ if and only if $\widetilde{n_{\phi}} \in C O P$, i.e. $\widetilde{n_{\phi}}$ is "constant on (Gleason) parts" in $\mathfrak{M} \backslash \mathbb{D}$. Note that this happens, for example, whenever $\phi$ is such that $\widetilde{n_{\phi}} \in C(\overline{\mathbb{D}})$, but as far as we know, it is not known which holomorphic maps $\phi: \mathbb{D} \rightarrow \mathbb{D}$ would satisfy this. At this point, we are also not aware of any results which would describe the non-constant, holomorphic maps $\phi: \mathbb{D} \rightarrow \mathbb{D}$ for which $\widetilde{n_{\phi}} \in C O P$, an we believe that this is an interesting problem for its own sake. Thus, we ask the following.

Question: For which holomorphic non-constant maps $\phi: \mathbb{D} \rightarrow \mathbb{D}$ does $\widetilde{n_{\phi}} \in C(\overline{\mathbb{D}})$, or more generally, for which such $\phi$ does $\widetilde{n_{\phi}} \in C O P$ ?

Acknowledgement: Research supported in part by NSERC grant.

\section{References}

[1] J. Arazy, S.D. Fisher, J. Peetre, Möbius invariant function spaces, J. Reine Angew. Math. 363 (1985) 110 - 145.

[2] S. Axler, D. Zheng, Boundary behaviour of derivatives of analytic functions, Michigan Math. J. 39 (1992) 129 - 143.

[3] S. Axler, D. Zheng, Compact operators via the Berezin transform, Indiana Univ. Math. J. 47 (1998) 387 - 400.

[4] S. Axler, D. Zheng, The Berezin transform on the Toeplitz algebra, Studia Math. 127 (1998) 113 - 136.

[5] P. Ghatage, M. Tjani, Closed range composition operators on Hilbert function spaces, J. Math. Anal. Appl. 431 (2015) 841 866.

[6] P. Gorkin, Functions not vanishing on trivial Gleason parts of Douglas algebras, Proc. Amer. Math. Soc. 104 (1998) 1086 1090. 
[7] M. Jovović, B. MacCluer, Composition operators on Dirichlet spaces, Acta Sci. Math. 63 (1997) 229 - 247.

[8] D. Luecking, Inequalities on Bergman spaces, Illinois J. Math. 25 (1981) 1 - 11.

[9] D. Luecking, Characterization of certain classes of Hankel operators on the Bergman spaces, J. Funct. Anal. 110 (1992) 247 271.

[10] D. Luecking, Bounded composition operators with closed range on the Dirichlet space, Proc. Amer. Math. Soc. 128 (2000) 1109 - 1116

[11] G. McDonald, C. Sundberg, Toeplitz operators on the disc, Indiana Univ. Math. J. 28 (1979) 595 - 611.

[12] K. Stroethoff, D. Zheng, Toeplitz and Hankel operators on Bergman spaces, Trans. Amer. Math. Soc. 329 (1992) 773 - 794.

[13] X. Zhao, D. Zheng, Invertibility of Toeplitz operators via Berezin Transforms, J. Oper. Th. 75 (2016) 475 - 495.

[14] K. Zhu, VMO, ESV, and Toeplitz operators on the Bergman space, Trans. Amer. Math. Soc. 302 (1987) 617 - 646.

[15] K. Zhu, Operator Theory in Function Spaces, Marcel Dekker, New York 1990.

[16] N. Zorboska, Composition operators on weighted Dirichlet spaces, Proc. Amer. Math. Soc. 126 (1998) 2013 - 2023.

[17] N. Zorboska, Toeplitz operators with BMO symbols and the Berezin transform, Int. J. Math. Sci. 2003, no. 46, 2929 - 2945.

[18] N. Zorboska, Closed range type properties of Topelitz operators on the Bergman space and the Berezin transform, Complex Anal. Oper. Theory, DOI 10.1007/s11785-019-00949-4 